\section{Identification of the amino acid labionin and its desulfurised derivative in the type-III lantibiotic LabA2 by means of GC/MS $\dagger$}

\author{
Received 18th March 2011, Accepted 19th April 2011 \\ DOI: $10.1039 / \mathrm{c} 1 \mathrm{cc11573a}$
}

Alexander Pesic, $\ddagger$ Maik Henkel $\ddagger$ and Roderich D. Süssmuth*

A GC-MS method for the rapid and unambiguous identification of the amino acid labionin (Lab) occurring in type-III lantibiotics is presented. This method will constitute a valuable tool for the characterisation and structure elucidation of labyrinthopeptins and their differentiation from lanthionine-type lantibiotics.

Lantibiotics are ribosomally synthesised peptide antibiotics containing the thioether amino acids $(2 S, 6 R)$-meso-lanthionine (Lan, 1) and/or (2S,3S,6R)-3-methyl-lanthionine (MeLan, 2). ${ }^{1}$ As further structural modifications lantibiotics may contain $\alpha, \beta$-unsaturated amino acids such as 2,3-didehydroalanine (Dha, 3) and 2,3-didehydroaminobutyric acid (Dhb, 4). Lantibiotics are produced by a wide range of Gram-positive bacteria, e.g. Staphylococci and Lactococci, and mostly have antimicrobial activity against other Gram-positive bacteria.

Recently, we identified a new structural type of lantibiotics, named labyrinthopeptins, which are produced by the Actinomycete Actinomadura namibiensis DSM6313. ${ }^{2}$ The structure model of Labyrinthopeptin A2 (LabA2, 6a) showed the presence of an unusual $\alpha, \alpha$-disubstituted amino acid, named labionin (Lab, 5). From X-ray data it was deduced that Lab (5) has $(2 S, 4 S, 8 R)$-configuration. Lab (5) can be structurally considered as a triamino triacid consisting of a basic lanthionine motif, which is further extended at the quaternary $\mathrm{C}$ - $\alpha$-atom by a methylene bridge and an $\alpha$-amino acyl residue, thus enabling the formation of two ring systems within a peptide chain. From the sequence of labyrinthopeptin prepropeptides it was deduced that a Ser/Ser/Cys motif is the precursor of Lab (5). Searches in databases containing genomic data showed that gene clusters with similar architecture and high sequence homology to the labyrinthopeptin gene cluster are widespread among Actinomycetes, e.g. Streptomyces coelicolor, S. avermitilis, and Saccharopolyspora erythraea. Based on those similarities we assume that they are producers of labyrinthopeptin-type lantibiotics. It is particularly interesting that their lantibiotic precursor peptides also contain the Ser/Ser/Cys motif, which could be a precursor of the Lab (5) motif (Fig. 1).

Technische Universität Berlin, Institut für Chemie, Strasse des 17, Juni 124, 10623 Berlin, Germany.

E-mail: suessmuth@chem.tu-berlin.de; Fax: +49 30314 79651;

Tel: +493031478774

$\dagger$ Electronic supplementary information (ESI) available: MS data and synthesis of amino acid standard. See DOI: $10.1039 / \mathrm{clcc1} 1573 \mathrm{a}$

$\ddagger$ Both authors contributed equally to this work.

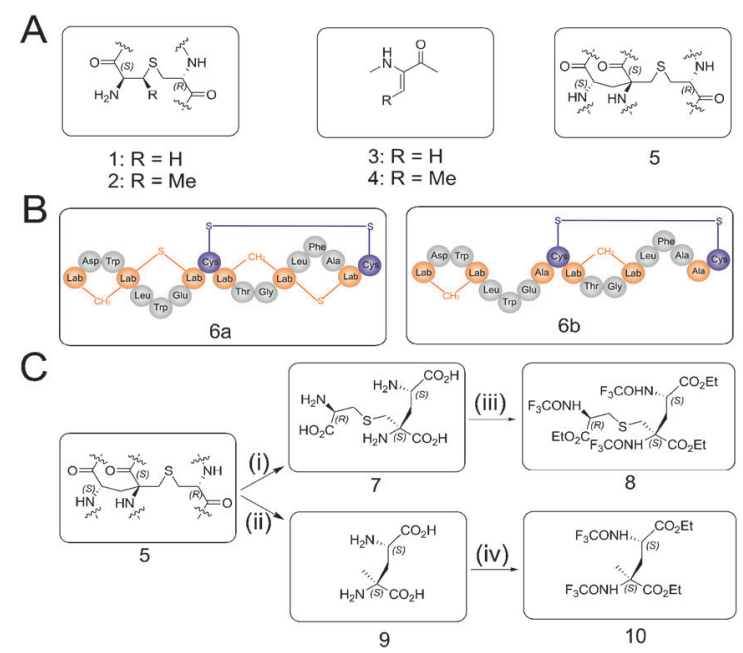

Fig. 1 (A) Structures of the amino acids Lan (1), MeLan (2), Dha (3) and Dhb (4) characteristic of lantibiotics. Amino acid Lab (5) in LabA2 is characteristic for recently discovered type-III lantibiotics of the labyrinthopeptin-type. (B) Schemes of LabA2 (6a) and desulfoLabA2 (6b). (C) Scheme of hydrolysis and microderivatisation for subsequent GC/MS analysis of Lab (7) and $\alpha$-methyl-2,4-diaminoglutaric acid (Dmg, 9): $N$-trifluoroacetyl/ethyl ester derivatisation to 8 and 10 by (i) $6 \mathrm{~N} \mathrm{HCl} ; 24 \mathrm{~h} ; 110{ }^{\circ} \mathrm{C}$, (ii) reductive desulfurisation with $\mathrm{NiCl}_{2} / \mathrm{NaBH}_{4} ; 6 \mathrm{~N} \mathrm{HCl} ; 24 \mathrm{~h} ; 110^{\circ} \mathrm{C}$, (iii, iv) AcCl; EtOH; TFAA.

However, structure elucidation of labyrinthopeptins as well as of related type-III lantibiotics from abovementioned bacterial producers is challenging. The high cyclisation degree of LabA2 (6a) poses significant problems to mass spectrometric sequencing. This is aggravated by two possible alternative isobaric posttranslational modifications Dha + Lan $(\mathbf{3}+\mathbf{1})$ and Lab (5) in type-III lantibiotics. Furthermore, structure elucidation of labyrinthopeptins by NMR spectroscopy is hampered by aggregation-prone properties. ${ }^{2}$ In this context GC-MS appears as a very useful technique for the detection, quantitation and structure elucidation of building blocks or modifications of peptidic natural products, e.g. amino acids, carbohydrates and lipids. Amino acids are commonly derivatised as $\mathrm{N}$-trifluoroacetyl or $\mathrm{N}$-pentafluorophenyl amino acid esters. ${ }^{3}$ The analytics of building blocks with higher molecular mass by GC-MS however is aggravated by a decrease in volatility of the derivatives with increasing molecular mass. Polar functional groups require additional 
derivatisations exhausting the limits of GC-MS as an analytical method.

The identification of the amino acid Lan (1) and MeLan (2) in lantibiotics by means of GC/MS is known for several years, ${ }^{4}$ in contrast, no analytical protocols were available for the detection of recently discovered triamino triacid Lab (7). In this contribution for the first time we report on the identification of the amino acid Lab (7) and its desulfurised derivative acid $\alpha$-methyl-2,4-diaminoglutaric acid (Dmg, 9) from lantibiotics of the labyrinthopeptin-type by means of GC-PCI-MS (PCI = Positive Chemical Ionisation). This achievement provides rapid access to an unambiguous identification of Lab (7) and analogues and will ultimately assist in the assignment of unknown lantibiotics to distinct lantibiotic classes.

We sought to develop robust protocols for a differentiation between Lan/MeLan (1/2) and Lab (5). Therefore, total hydrolysis of the lantibiotic nisin as a model system was performed and the hydrolysate was derivatised as $N$-trifluoroacetyl amino acid ethylesters. Detection of Lan/MeLan (1/2) by means of GC-PCI-MS in Nisin (ESI†, 25, Fig. S7 and S10) was established according to previous reports. ${ }^{4}$ In the same manner we subsequently performed total hydrolysis of LabA2 (6a) and derivatisation of the hydrolysate. As expected, the signals of the corresponding $N$-trifluoroacetyl/ethyl esters could be identified and assigned to Ala, Gly, Thr, Leu, Asp, Glu, Phe and Trp with the exception of (Cys) 2 (Fig. 2A). Additionally the GC-MS chromatogram revealed a signal at $R_{\mathrm{t}}=36.3 \mathrm{~min}$ (Fig. 2B) assigned to derivatised Lab (8). This was corroborated by indicative molecular ions $[\mathrm{M}+\mathrm{H}]^{+}=$ $668 \mathrm{Da}$ and $\left[\mathrm{M}+\mathrm{C}_{2} \mathrm{H}_{5}\right]^{+}=696 \mathrm{Da}$ of derivatised Lab (8) under mild GC-PCI-MS conditions (Fig. 2C) and assignment of characteristic ion fragments (Fig. S11, ESI $\dagger$ ). These ions

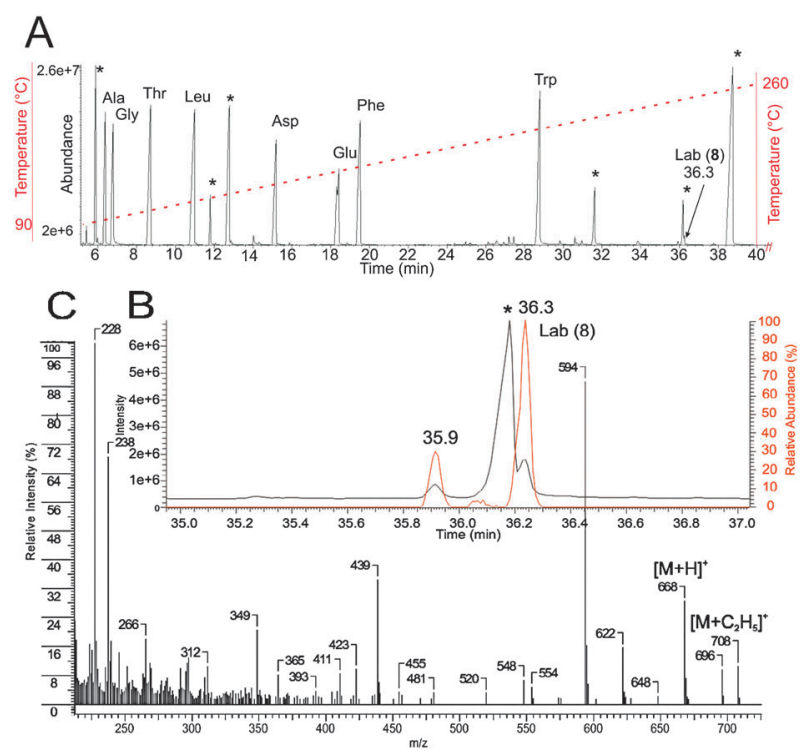

Fig. 2 (A) GC-MS chromatogram of the hydrolysate of LabA2 (6a) $(6 \mathrm{~N} \mathrm{HCl}, 24 \mathrm{~h})$ after $N$-trifluoroacetyl/ethylester derivatisation. (B) Enlarged section of (A) Lab (8) (TIC in black and normalised $\mathrm{XIC}[\mathrm{M}+\mathrm{H}]^{+}=668 \mathrm{Da}$ in red). (C) PCI mass spectrum of $N$-trifluoroacetyl/ethylester of $\mathrm{Lab}(\mathbf{8})$ at $R_{\mathrm{t}}=36.3 \mathrm{~min}$. Peaks marked with asterisk $(*)$ are unidentified byproducts. show characteristic fragmentations, which strongly depend on the structure of analysed $N$-trifluoroacetyl amino acid ester. ${ }^{5}$ The $\mathrm{XIC}$ for $[\mathrm{M}+\mathrm{H}]^{+}=668 \mathrm{Da}$ (Fig. 2B) revealed also a peak at $R_{\mathrm{t}}=35.9$ min with identical mass spectrum (data not shown) as for $R_{\mathrm{t}}=36.3 \mathrm{~min}$ (Fig. 2C), which suggests epimerization of Lab as a likely explanation for this observation.

The relative signal intensities in the GC-MS compared to other amino acid constituents were significantly reduced and several other compounds eluting from the column could not be assigned (Fig. 2A).

In order to give additional proof on the occurrence and the assignment of Lab (7), degradation of LabA2 (6a) by desulfurisation of thioethers was applied as previously reported for subtilosin $\mathrm{A}^{6}$ using $\mathrm{NiCl}_{2} / \mathrm{NaBH}_{4}$ treatment under retention of the stereochemistry. ${ }^{7}$ Progress of desulfurisation was monitored by LC-ESI-MS and the reaction was completed within 24 hours. Fig. 3 shows the HR-ESI(+)-orbitrap mass spectra of LabA2 (6a, in black) before $\left[(\mathrm{M}+2 \mathrm{H})^{2+}=962 \mathrm{Da}\right]$ and after $(6 \mathbf{b}$, in red $)$ desulfurisation $\left[(\mathrm{M}+2 \mathrm{H})^{2+}=931 \mathrm{Da}\right]$ corresponding to a formal loss of two sulfur atoms and formal addition of four hydrogen atoms. Under the reaction conditions applied, the integrity of cystine remained untouched.

From the progress of the desulfurisation monitored by LC-MS transformation of Lab (7) into $\alpha$-methyl-2,4-diaminoglutaric acid (Dmg, 9) and Ala was expected. Conversion of the hydrolysate of desulfo-LabA2 (6b) into the $N$-trifluoroacetyl/ethyl esters and subsequent GC-MS analytics gave a new signal at $R_{\mathrm{t}}=20.9$ min (Fig. 4A and 5B) whereas the previous signal from derivatised Lab $(\mathbf{8})$ had disappeared.

Trp was not detected after desulfurisation (Fig. 4A) of LabA2 (6a) whereas several other compounds eluted from the column which could not be identified. Nevertheless, characteristic molecular ions for Dmg derivative (10) $\left(R_{\mathrm{t}}=\right.$ $20.9 \mathrm{~min}),[\mathrm{M}+\mathrm{H}]^{+}=425 \mathrm{Da}$ and $\left[\mathrm{M}+\mathrm{C}_{2} \mathrm{H}_{5}\right]^{+}=453 \mathrm{Da}$ shown in Fig. 4B and characteristic mass fragments were assigned (Fig. S12, ESI $\dagger$ ).

Our intention was to independently verify the identity of Dmg (9) by synthesis of the racemate according to Scheme 1. Synthetic access was obtained by Claisen-type rearrangement reaction of Cbz-L-alanine-allylester (13) to Cbz- $\alpha$-allyl-alanine as the central step. ${ }^{8}$ Subsequent to this step a Sharpless dihydroxylation ${ }^{9}$ of the allyl moiety (15) and selective protection of the primary alcohol with TBDMS $( \pm \mathbf{1 7} \mathbf{a} / \mathbf{b})$ was performed.

By a Mitsunobu reaction an azide group was introduced and further steps comprised TBDMS deprotection, oxidation of the resulting alcohol, removal of protecting groups, hydrogenolysis and $N$-trifluoroacetyl/ethylester derivatisation which

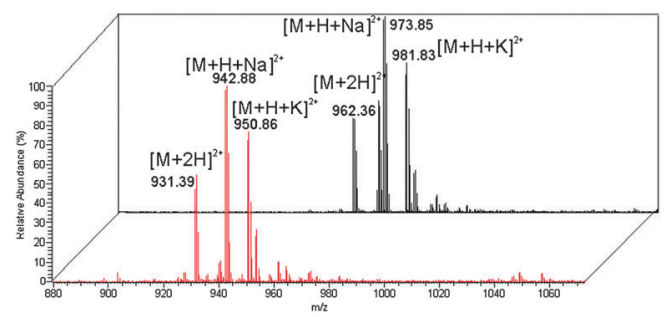

Fig. 3 HR-ESI(+)-orbitrap mass spectra of LabA2 before desulfurisation (black, 6a) $\left[(\mathrm{M}+2 \mathrm{H})^{2+}=962 \mathrm{Da}\right]$ and after desulfurisation $(\mathrm{red}, \mathbf{6 b})\left[(\mathrm{M}+2 \mathrm{H})^{2+}=931 \mathrm{Da}\right]$. 

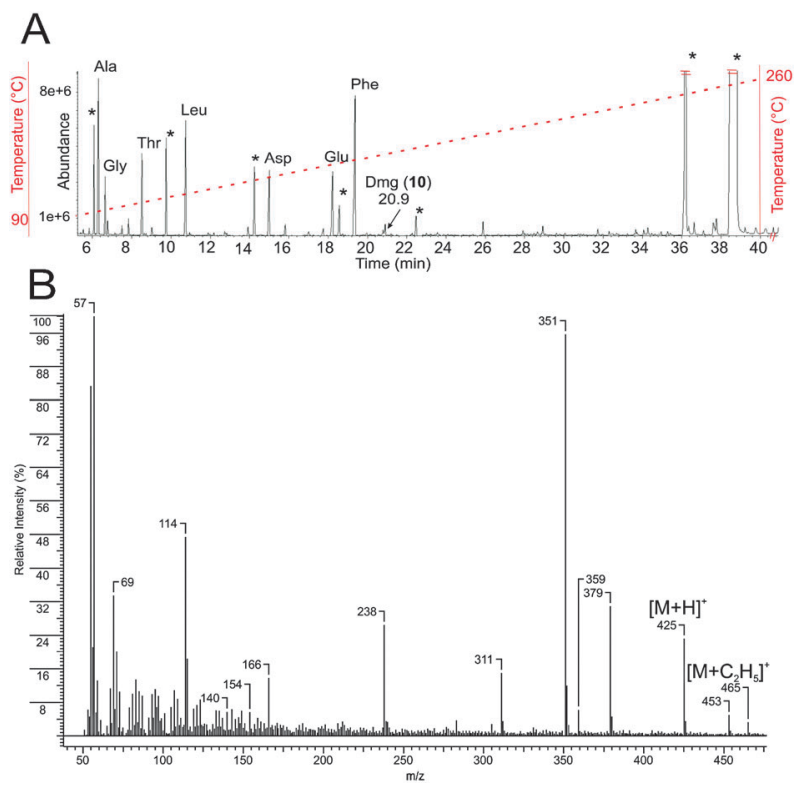

Fig. 4 (A) GC-MS chromatogram of the desulfo-LabA2 (6b) hydrolysate after $N$-trifluoroacetyl/ethylester derivatisation. (B) PCI mass spectrum of the $N$-trifluoracetyl/ethylester of Dmg (10) at $R_{\mathrm{t}}=$ 20.9 min.

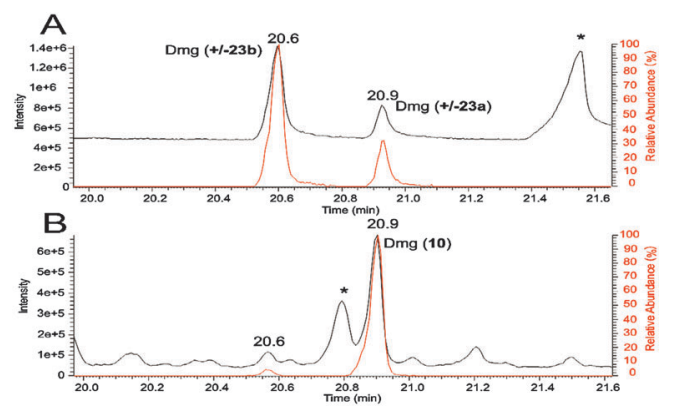

Fig. 5 GC-MS analytics. (A) Synthetic diastereomers of Dmg $( \pm \mathbf{2 3 a} / \mathbf{b})$ (TIC in black and normalised XIC $[\mathrm{M}+\mathrm{H}]^{+}=425 \mathrm{Da}$ in red). (B) Dmg (10) from desulfurised hydrolysate of LabA2 (6b) (TIC in black and normalised XIC $[\mathrm{M}+\mathrm{H}]^{+}=425 \mathrm{Da}$ in red). Peaks marked with asterisk $(*)$ are undefined byproducts.

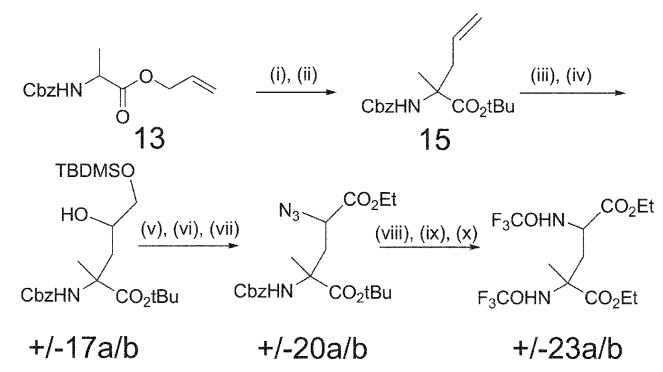

Scheme 1 Synthesis of Dmg $( \pm \mathbf{2 3 a} / \mathbf{b})$ as a standard for assignment of Lab derivatives after desulfurisation, hydrolysis and $\mathrm{N}$-trifluoroacetyl/ ethylester derivatisation. (i) $\mathrm{LDA}$; $\mathrm{ZnCl}_{2}$, (ii) $\mathrm{BF}_{3}{ }^{*} \mathrm{OEt}$; TBTA, (iii) AD-Mix- $\beta$, (iv) TBDMSCl; imidazol, (v) $\mathrm{PPh}_{3}$; DEAD; DPPA, (vi) TBAF, (vii) TEMPO; $\mathrm{KBr}$; $\mathrm{NaOCl}$, (viii) TFA; TES, (ix) $\mathrm{Pd} / \mathrm{C}$; $\mathrm{H}_{2}$, (x) AcCl; EtOH; TFAA.

finally led to synthetic Dmg $( \pm \mathbf{2 3} \mathbf{a} / \mathbf{b})$. The stereochemistry of synthetic Dmg diastereomers $( \pm \mathbf{2 3 a} / \mathbf{b})$ was afterwards deduced by comparison of the retention time of Dmg (10).
Fig. 5(A) shows the GC-MS analysis of synthetic $\pm \mathbf{2 3 a} / \mathbf{b}$. The gradient allowed to separate the diastereomeric pairs $\pm \mathbf{2 3 b}$ at $R_{\mathrm{t}}=20.6 \mathrm{~min}$ and $\pm \mathbf{2 3 a}$ at $R_{\mathrm{t}}=20.9 \mathrm{~min}$, respectively. As expected both pairs showed identical fragmentations patterns under PCI-MS conditions $( \pm \mathbf{2 3 a} / \mathbf{b}$, Fig. S8, ESI $\dagger$ ) compared to the natural product derived compound Dmg (10). The identity in retention time (Fig. 5A and B) and fragmentation patterns of synthetic reference compounds \pm 23a unequivocally proved the identity of this amino acid as Dmg (10, Fig. 4B and 5B).

The derivatisation protocol combined with GC-MS analysis developed for Lab (7)/Dmg (9) was applied to the analysis of Labyrinthopeptin A1 (LabA1) and desulfurised LabA1 (ESI $\dagger, \mathbf{2 4 a} / \mathbf{b}$, Fig. S5 and S13), respectively. LabA1 is a member of the labyrinthopeptin family, for which the structure has mainly been derived from genomic data of the biosynthesis gene cluster. ${ }^{2}$ In accordance to the analytics of LabA2 $(\mathbf{6 a} / \mathbf{b})$ the GC-MS data of LabA1 (ESI $\dagger, 24 \mathbf{a} / \mathbf{b}$, Fig. S5, S9, and S13) also could unambiguously prove the presence of Lab (7) and Dmg (9) for the first time.

In summary, we have established a protocol for the detection of the unusual triamino triacid Lab (7) by GC/MS from LabA2 hydrolysates. Furthermore, the above strategy combined with $\mathrm{NiCl}_{2} / \mathrm{NaBH}_{4}$-mediated desulfurisation of Lab-containing type-III lantibiotics can be independently applied for GC-MS analysis of derivative Dmg (9). Subsequently, this protocol was successfully applied to the detection of Lab (7) and Dmg (9) from labyrinthopeptin LabA1 and desulfurised LabAl (ESI†, 24a/b, Fig. S13), respectively. The detection of Lab (7) and Dmg (9) complements the presently available methods for the analytics of lantibiotics. Our future attempts will extend on other type-III lantibiotics to prove the occurrence of Lab (7) (ESI $\dagger, 24 a$, Fig. S13) in these lantibiotics and we hypothesise that Lab (7) could be considered as a common structural motif characteristic for many type-III lantibiotics.

The work was supported by a research grant from the Deutsche Forschungsgemeinschaft (SU239/8-1).

\section{Notes and references}

1 J. M. Willey and W. A. van der Donk, Annu. Rev. Microbiol., 2007, 61, 447.

2 (a) K. Meindl, T. Schmiederer, K. Schneider, A. Reicke, D. Butz, S. Keller, G. Nicholson, H. Gühring, L. Vértesy, J. Wink, H. Hoffmann, M. Broenstrup, G. M. Sheldrick and R. D. Süssmuth, Angew. Chem., Int. Ed., 2010, 49, 1151; (b) W. M. Müller, T. Schmiederer, P. Ensle and R. D. Süssmuth, Angew. Chem., Int. Ed., 2010, 49, 2436.

3 H. Frank, G. J. Nicholson and E. Bayer, J. Chromatogr., A, 1978, 167, 187.

4 (a) E. Kösters, H. Allgaier, G. Jung and E. Bayer, Chromatographia, 1984, 18, 287; (b) B. Li, D. Sher, L. Kely, Y. Shi, K. Huang, P. J. Knerr, I. Joewono, D. Rusch, S. W. Chisholm and W. A. van der Donk, Proc. Natl. Acad. Sci. U. S. A., 2010, 107, 10430.

5 A. Prox and J. Schmid, Org. Mass Spectrom., 1969, 2, 105.

6 E. K. Kawulka, T. Sprules, C. M. Diper, R. M. Whittal, T. R. McKay, P. Mercier, P. Zuber and J. C. Vederas, Biochemistry, 2004, 43, 3385.

7 T. G. Back, D. L. Baron and K. J. Yang, J. Org. Chem., 1993, 58, 2407.

8 U. Katzmaier and S. Maier, Tetrahedron, 1996, 52, 941.

9 H. C. Kolb, M. S. van Nieuwenhze and K. B. Sharpless, Chem. Rev., 1994, 94, 2483. 